\title{
Anomalous Specific-Heat Jump in a Two-Component Ultracold Fermi Gas
}

\author{
Armen Sedrakian, ${ }^{1}$ Herbert Müther, ${ }^{1}$ and Artur Polls ${ }^{2}$ \\ ${ }^{1}$ Institute for Theoretical Physics, Tübingen University, D-72076 Tübingen, Germany \\ ${ }^{2}$ Departament d'Estructura i Constituents de la Matèria, Universitat de Barcelona, E-08028 Barcelona, Spain
}

(Received 3 May 2006; published 4 October 2006)

\begin{abstract}
The thermodynamic functions of a Fermi gas with spin population imbalance are studied in the temperature-asymmetry plane in the BCS limit. The low-temperature domain is characterized by an anomalous enhancement of the entropy and the specific heat above their values in the unpaired state, decrease of the gap and eventual unpairing phase transition as the temperature is lowered. The unpairing phase transition induces a second jump in the specific heat, which can be measured in calorimetric experiments. While the superfluid is unstable against a supercurrent carrying state, it may sustain a metastable state if cooled adiabatically down from the stable high-temperature domain. In the latter domain the temperature dependence of the gap and related functions is analogous to the predictions of the BCS theory.
\end{abstract}

DOI: 10.1103/PhysRevLett.97.140404

Recent experiments [1,2] on ultracold dilute gases of fermionic atoms trapped an unequal number of fermions in two different hyperfine states. These experiments started addressing some of the long-standing problems in the theory of asymmetric superconductors (ASCs) that are of interest in a variety of fields including metallic superconductors [3,4], nuclear systems [5-7] and high density QCD [8-11]. The unprecedented control over the many-body systems achieved in the experiments with ultracold dilute fermions combined with the possibility of tuning the interactions via the Feshbach resonance mechanism provide for the first time a realistic perspective of testing the predictions of the theories of ASC in the context of dilute fermionic systems. The realizations of various phases of ASC of dilute fermions have been intensively studied on the theoretical front; the simplest realizations are the isotropic, homogeneous phases that are characterized either by a Zeeman splitting of Fermi levels [12-14] or by pairing between light and heavy fermions $[15,16]$. At large asymmetries the phases with broken space symmetries [17-24] and the mixed phases $[25,26]$ become energetically more favorable. Alternatives include pairing in higher angular momentum states $[27,28]$. Finite-size and trap geometry introduce an additional complication to the problem and may qualitatively affect the comparison between the theory and experiment $[29,30]$. A number of related problems of interest are the nature of phase transitions between the various phases and their relation to the topology of Fermi surfaces [31,32] as well as the features of the BCS-BEC crossover [33-35] under population imbalance.

The population asymmetry in ASC can be characterized either in terms of the difference (mismatch) in the chemical potentials or the difference in the densities of the species. The first case arises when the "chemical" equilibrium between populations admits transmutation between the different spin states, as, e.g., under the equilibrium with respect to the weak interactions in cold dense hadronic or quark matter. We shall specify our discussion from the outset to the second case and assume that the total number
PACS numbers: 05.30.Fk, 03.75.Hh, 03.75.Ss, 74.20.Fg

of fermions is fixed and the asymmetry is maintained with respect to the number densities of different species, as is the case in the experiments on ultracold fermions.

The aim of this Letter is the study of the temperatureasymmetry phase diagram of an ultracold Fermi gas with pairing between two unequally populated hyperfine states in the BCS regime. We propose that calorimetric experiments, which are within the current experimental capabilities [36], can test the specific features of the phase diagram discussed below. The critical temperature of metallic superconductors, according to the Bardeen-CooperSchrieffer (BCS) theory, is given by $T_{c}=1.14 \omega_{D} e^{-1 / V}$, where $\omega_{D}$ is the Debye frequency and $V$ is the dimensionless interaction. The parameters in this equation can be determined from a single calorimetric experiment; $T_{c}$ is determined from the position of the jump in the specific heat, and $\omega_{D}$ from the slope of the specific heat over $T^{3}$ in the limit $T \rightarrow 0$. The case of ASC is complicated by the fact that the asymmetry leads to a loss of coherence at low temperatures and the critical temperature and pairing gap become complicated functions of spin imbalance. This Letter studies the impact of the asymmetry induced decoherence on the thermodynamics of ultracold gases; in particular, we show that two jumps in the specific heat of ASC are possible if the asymmetry is large, albeit the second anomalous jump occurs within the temperature domain where the superfluid is in a metastable state. The anomalous jump in the specific heat is a manifestation of the reentrance effect, i.e., the restoration of paircorrelations at finite temperatures $T \leq T_{c}$ [5,37,38]. Below, we shall confine ourselves to the case of infinitely extended systems. Since the experiments are carried out in finite geometries, finite-size corrections need to be taken into account in a more complete analysis. Recent experiments $[1,2]$ which have measured the density profiles of trapped gases with population imbalance demonstrate the importance of these effects in determining the ground state structure of the condensate $[29,30]$. Related work on finite temperature phase diagram of ASC appeared in 
Refs. [39,40] while our work was in preparation or revision.

We approximate the pairing interaction by a zero-range force, which is characterized by the $s$-wave scattering length $a_{S}$. Our discussion is specific to the case where two hyperfine states of ${ }^{6} \mathrm{Li}$ are populated (the scattering length in units of the Bohr radius is $a_{S} / a_{B}=-2160$, but can be varied at will via Feshbach resonance mechanism). The fermion masses are assumed to be equal; the extension to the case of unequal masses (as would be the case in the mixtures of ${ }^{6} \mathrm{Li}$ and ${ }^{40} \mathrm{~K}$ ) is straightforward.

We consider a uniform gas of fermionic atoms in two hyperfine states (spins) labeled as $\uparrow$ and $\downarrow$; the interaction Hamiltonian is

$$
\hat{H}_{\mathrm{int}}=-V \sum_{\alpha \beta} \int d^{3} x \hat{\psi}_{\alpha}^{\dagger}(\boldsymbol{x}) \hat{\psi}_{\beta}^{\dagger}(\boldsymbol{x}) \hat{\psi}_{\beta}(\boldsymbol{x}) \hat{\psi}_{\alpha}(\boldsymbol{x}),
$$

where $\hat{\psi}_{\alpha}^{\dagger}(\boldsymbol{x})$ and $\hat{\psi}_{\alpha}(\boldsymbol{x})$ are the creation and annihilation operators of a state at the space point specified by the position vector $\boldsymbol{x}$ and spin $\alpha(=\uparrow, \downarrow)$ and $V$ is the two-body bare contact interaction. The normal and anomalous propagators are [19] $G_{\uparrow \downarrow}(p)=\omega+\mathcal{E}_{S} \pm \mathcal{E}_{A} / D, \quad F^{\dagger}(p)=$ $\Delta^{\dagger} / D$, where $D \equiv\left(\omega-\mathcal{E}_{A}\right)^{2}-\mathcal{E}_{S}^{2}-\Delta^{2}$ and $\mathcal{E}_{S}=$ $\left(\varepsilon_{p \uparrow}+\varepsilon_{p \downarrow}\right) / 2$ and $\mathcal{E}_{A}=\left(\varepsilon_{p \uparrow}-\varepsilon_{p \downarrow}\right) / 2$ are, respectively, the parts of the quasiparticle spectrum which are symmetric and antisymmetric under time-reversal operation. Here $\epsilon_{p \uparrow}$ and $\epsilon_{p \downarrow}$ are the single particle energies in states $\uparrow$ and $\downarrow$. The dispersion relation of the quasiparticles in the paired state is $\omega_{\uparrow / \downarrow}=\mathcal{E}_{A} \pm \sqrt{\mathcal{E}_{S}^{2}+\Delta^{2}}$. The limit $\mathcal{E}_{A} \rightarrow 0$ corresponds to the case of equal number of spin-up and spindown particles. The explicit form of the symmetric and anti-

symmetric (under time-reversal) parts of the quasiparticle spectrum are $\mathcal{E}_{S}=\left(p^{2} / m-\mu_{\uparrow}^{*}-\mu_{\downarrow}^{*}\right) / 2$ and $\mathcal{E}_{A}=\left(-\mu_{\uparrow}^{*}+\right.$ $\left.\mu_{\downarrow}^{*}\right) / 2$, where $m$ is the atom's bare mass, $\boldsymbol{p}$ is the relative momentum of fermions bound in a Cooper pair in the center-of-mass frame at rest, the effective chemical potentials $\mu_{\uparrow / \downarrow}^{*}=\mu_{\uparrow / \downarrow}-\Sigma_{\downarrow / \uparrow}$ include the constant shift due to the self-energy $\Sigma_{\uparrow / \downarrow}=T_{0} \rho_{\downarrow / \uparrow}$, where $T_{0}$ is the two-body scattering $T$ matrix. The gap equation is

$$
\frac{2}{U_{0}}=\int_{0}^{\Lambda} \frac{1}{\sqrt{\mathcal{E}_{S}^{2}(p)+\Delta^{2}}}\left[f\left(\omega_{\uparrow}\right)-f\left(\omega_{\downarrow}\right)\right] \frac{p^{2} d p}{(2 \pi)^{2}},
$$

where $U_{0}$ is the strength of a contact interaction and $\Lambda$ is an ultraviolet cutoff (for details see Ref. [19]). The occupation probabilities of species are given by

$$
n_{\uparrow / \downarrow}(\boldsymbol{p})=u_{\boldsymbol{p}}^{2}\left[f\left(\omega_{\uparrow / \downarrow}\right)-f\left(-\omega_{\downarrow / \uparrow}\right)\right]+f\left(-\omega_{\downarrow / \uparrow}\right),
$$

where $u_{p}^{2}=1 / 2+\mathcal{E}_{S} /\left(2 \sqrt{\mathcal{E}_{S}^{2}+\Delta^{2}}\right)$; these are normalized to the densities of species $\rho_{\uparrow / \downarrow}=\sum_{p} n_{\uparrow / \downarrow}(\boldsymbol{p})$. The free energy is $\mathcal{F}=\sum_{p}\left(\epsilon_{p \uparrow} n_{p, \uparrow}+\epsilon_{p \downarrow} n_{p, \downarrow}\right)-U_{0}^{-1} \Delta^{2}-T S_{S}$. The free energy of the normal state follows by setting in this expressions $\Delta=0$. The entropy of ASC is defined in terms of the temperature derivative of the free energy $S_{S}=$ $-\partial \mathcal{F} / \partial T$. The specific heat follows as $C_{V}=$ $T\left(\partial S_{S} / \partial T\right)=-T\left(\partial^{2} \mathcal{F} / \partial T^{2}\right)$.

The local stability requires that the free energy is a convex function of the appropriate variables and it has been established that homogeneous ASC could become unstable in this sense $[16,24,25,32,33,35,39,41,42]$. Specifically, (A) the system is unstable against phase separation unless the curvature matrix $\chi_{i j}=\partial^{2} \mathcal{F}_{\mathcal{S}} / \partial \mu_{i} \partial \mu_{j}$ is positive definite. This implies that either the eigenvalues $\lambda_{1,2}=\operatorname{Tr} \chi_{i j} / 2 \pm \sqrt{\left(\operatorname{Tr} \chi_{i j}\right)^{2}-4 \operatorname{Det} \chi_{i j}} / 2 \geq 0$ or, equivalently, $\chi_{\Uparrow \uparrow}>0$, $\operatorname{det} \chi_{i j}>0$ (Sylvester criterion). Further, (B) the condition $\partial^{2} \mathcal{F}_{\mathcal{S}} / \partial \Delta^{2}>0$ needs to be fulfilled. Finally, (C) the system may become unstable against spontaneous generation of currents [formation of the LarkinOvchinnikov-Fulde-Ferrell (LOFF) phase] when $\chi_{q}=$ $\partial^{2} \mathcal{F}_{\mathcal{S}} / \partial q^{2}<0$, where $q$ is the center-of-mass of momentum of a Cooper pair. The latter instability manifests itself in the negative superfluid density, $\rho_{s}=\left.m^{2} \chi(q)\right|_{q=0}$, and purely imaginary Meissner mass. For small quasiparticle velocity $\boldsymbol{u}$ the leading order contribution to the ratio of the superfluid to the total density is [43]

$$
\frac{\rho_{s}}{\rho}(T)=1-\frac{1}{\rho m} \int \frac{d p p^{4}}{6 \pi^{2}}\left[\left.\frac{\partial f\left(\omega_{\downarrow}\right)}{\partial \omega_{\downarrow}}\right|_{\boldsymbol{u}=0}+\left.\frac{\partial f\left(\omega_{\downarrow}\right)}{\partial \omega_{\uparrow}}\right|_{\boldsymbol{u}=0}\right] .
$$

We fix the density of ${ }^{6} \mathrm{Li}$ atoms at $\rho=3.8 \times 10^{12} \mathrm{~cm}^{-3}$, which in the case of a spatially uniform and spinsymmetric system at $T=0$ corresponds to the Fermi momentum $k_{F}=4.83 \times 10^{4} \mathrm{~cm}^{-1}$ and $k_{F} a_{S}=-0.558$. Since we work in the BCS regime the conditions (A) and (B) are fulfilled (in agreement with Ref. [39]). Figure 1 displays the $\Delta(T), \Delta \mathcal{F}(T)=\mathcal{F}_{N}-\mathcal{F}_{S}$ and $\rho_{s}(T) / \rho$ functions for constant asymmetry $\alpha=\left(\rho_{\uparrow}-\rho_{\downarrow}\right) /\left(\rho_{\uparrow}+\rho_{\downarrow}\right)$. The length and energy are measured below in units of $L=$ $10^{-4} \mathrm{~cm}$ and $e=10^{2} \mathrm{nK}$, unless explicitly specified. Near the critical temperature the asymptotic behavior of the pairing gap for $T \rightarrow T_{c}$ is described, to leading order in $(\Delta / T)^{2}$, by the BCS-type relation $\Delta(\alpha) \sim\left[T_{c}(\alpha)\left(T_{c}(\alpha)-\right.\right.$ $T)]^{1 / 2}$; i.e., the high-temperature portions of the $\Delta(T)$ curves are self-similar. The low-temperature BCS asymptotics $\Delta(T) \sim \Delta(0)-\sqrt{2 \pi T \Delta(0)} e^{-\Delta(0) / T}$ is qualitatively modified even at small asymmetries, since the gap decreases as $T \rightarrow 0$, instead of staying constant. At large asymmetries (e.g., $\alpha \geq 0.04$ ) the reentrance effect sets in: the gap is nonzero only in a finite domain of temperatures bounded by two critical temperatures. The physical origin of the upper critical temperature is analogous to the BCS case, where the pairing correlations are destroyed by thermal fluctuations. The lower critical temperature is due to the loss of coherence induced by the asymmetric population and is specific to ASC. At large asymmetries the temperature dependence of the gap is $\Delta(\alpha) \sim\left[T_{c}^{*}(\alpha) \times\right.$ $\left.\left(T-T_{c}^{*}(\alpha)\right)\right]^{1 / 2}$, where $T_{c}^{*}$ is the lower critical temperature. The free-energy plots (Fig. 1) reflect the temperature 


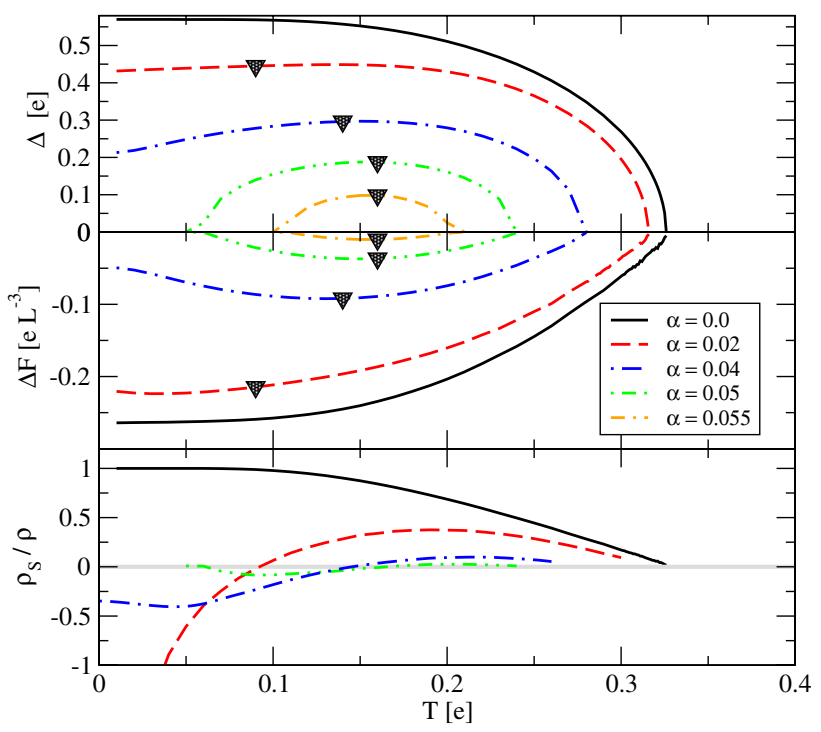

FIG. 1 (color online). Dependence of the pairing gap (upper panel), the free-energy difference (middle panel), and the superfluid density (lower panel) on the temperature for several values of the density asymmetry. The instability domain $\left(\rho_{s}<0\right)$ lies to the left of triangles.

dependence of the condensation energy, which scales as $\Delta^{2}(\alpha, T)$; the temperature dependence and asymptotics of $\Delta \mathcal{F}(T)$ is understandable in terms of this scaling. In the temperature domain where $\rho_{s}(T)<0$, the homogeneous ASC is metastable; there exists a lower extremum (perhaps minimum) of the free energy corresponding to the current carrying LOFF phase. Nevertheless if prepared at high enough (but $T<T_{c}$ ) temperature and cooled down adiabatically, the metastable phase can be sustained long enough to carry out measurements. The temperature dependence of the entropy and specific heat (more precisely $\left.C_{V} / T\right)$ is shown in Fig. 2. At $T \rightarrow T_{c}^{-}$the entropy scales linearly with temperature, $S_{S} \propto T-T_{c}$, with asymmetry dependent slope. In the low-temperature metastable region the superfluid entropy is anomalous, since its absolute magnitude is larger than the entropy of the normal state; i.e., the superfluid appears to be less ordered than the unpaired state. The temperature for the onset of anomalous regime $\left(S_{S}>S_{N}\right)$ coincides with that for the onset of instability within our numerical accuracy.

The ratio $\Delta C / T_{c}$, where $\Delta C=C_{S}-C_{N}$ is the jump in the specific heat at the critical temperature, depends only on the density of states and is a universal characteristic of a system. The jump itself is a characteristic feature of a second order phase transition that allows to locate $T_{c}$ experimentally. There is a second (anomalous) jump in the specific heat associated with the reentrance effect at the lower critical temperature $T_{c}^{*}$, which lies within the metastable domain. At $T \rightarrow T_{c}^{-}$the specific heat scales as $C_{V} \propto\left|T-T_{c}\right|$. Its low-temperature asymptotics differs dramatically from the predictions of BCS theory, where the superfluid "thermal inertia" is small compared to the

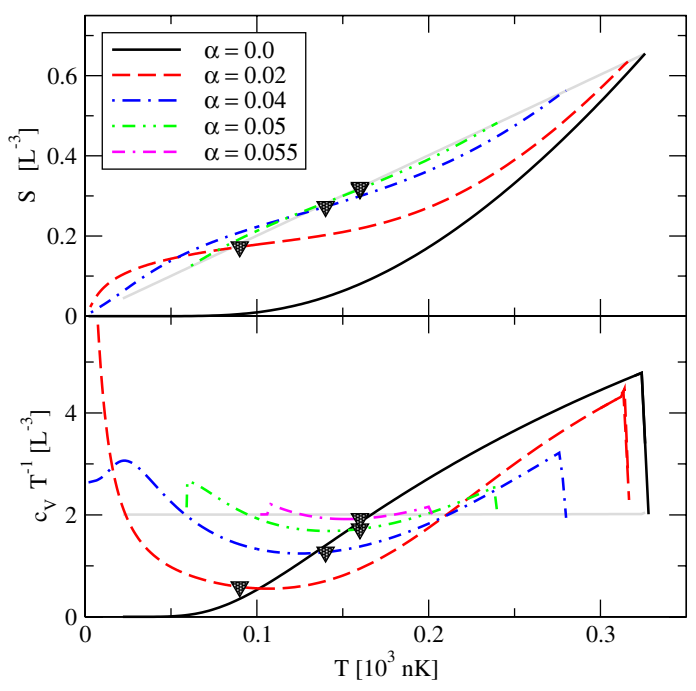

FIG. 2 (color online). Entropy (upper panel) and the heat capacity (lower panel) as a function of the temperature for several values of the density asymmetry. Note that for $\alpha=$ $0.02, c_{V}(T=0)=4.5$ is finite. The gray lines show the values in the normal state.

normal case. The specific heat of ASC is larger than that of the normal state at sufficiently low temperature for any asymmetry. Calorimetric experiments aimed at measuring the specific heat of ASC can locate the critical temperature and observe the reentrance effect through the second (anomalous) jump $\Delta C_{V}$, if the system can be maintained long enough in the metastable state.

The occupation probability of the majority and minority components are shown in Fig. 3 for the high- and lowtemperature regions of pairing. The remarkable difference

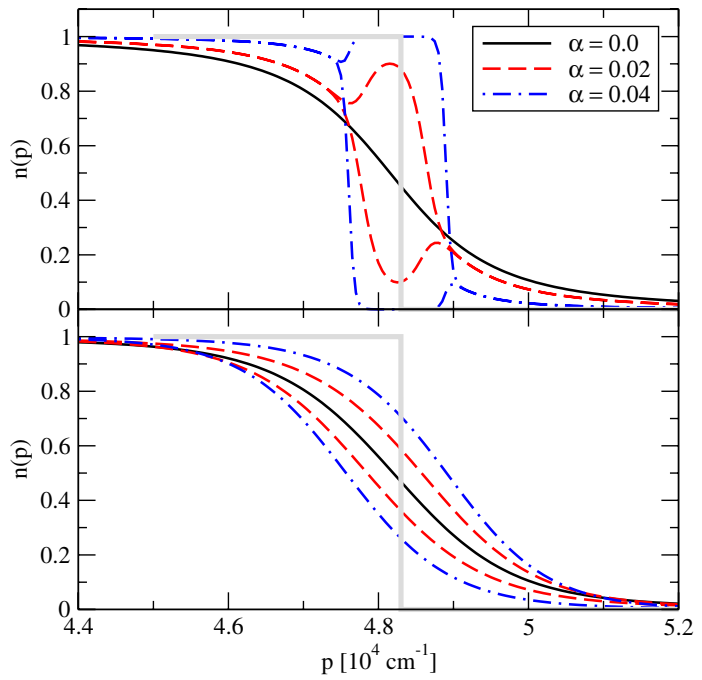

FIG. 3 (color online). Occupation probabilities of the majority and minority components as a function of the momentum for several asymmetries at temperatures $T=1 \mathrm{nK}$ (upper panel) and $T=25 \mathrm{nK}$ (lower panel). All lines correspond to stable states, except $\alpha \neq 0$ lines in the upper panel. The gray line shows the same for the unpaired symmetric state at $T=0$. 
between these two cases arises from the fact that in the low-temperature regime the minority component is excluded from the region around the Fermi momentum ("blocking region" [6] or "breach" $[15,16])$. The depletion is large for large asymmetries. In contrast, the hightemperature regime does not feature a depletion region and the occupation are smooth functions of the momenta for arbitrary asymmetries.

The finite range of interactions and finite size of the systems are not likely to modify the conclusions reached here. Indeed, reentrance behavior(s) have been predicted in paired nuclear systems which are characterized by complex finite range interactions $[5,37,44]$ and ultrasmall metallic grains, which contain a small number of fermions and a single (odd) unpaired particle [38].

We are grateful to Jorge Dukelsky, Jordi Mur-Petit, Peter Schuck, and Claus Zimmerman for useful interactions. This work was in part supported by the SFB 382 of the DFG (Germany) and Grants No. FIS2005-03142 (MEC, Spain and FEDER) and No. 2005SGR-00343 (Generalitat de Catalunya).

[1] M.W. Zwierlein, A. Schirotzek, C.H. Schunck, and W. Ketterle, Science 311, 492 (2006); Y. Shin, M. W. Zwierlein, C.H. Schunck, A. Schirotzek, and W. Ketterle, Phys. Rev. Lett. 97, 030401 (2006).

[2] G. B. Partridge, W. Li, R. I. Kamar, Y. Liao, and R. G. Hulet, Science 311, 503 (2006).

[3] A. I. Larkin and Y. N. Ovchinnikov, Zh. Eksp. Teor. Fiz. 47, 1139 (1964) [Sov. Phys. JETP 20, 762 ( 1965)].

[4] P. Fulde and R. A. Ferrell, Phys. Rev. 135, A550 (1964).

[5] A. Sedrakian and U. Lombardo, Phys. Rev. Lett. 84, 602 (2000).

[6] U. Lombardo, P. Nozières, P. Schuck, H.-J. Schulze, and A. Sedrakian, Phys. Rev. C 64, 064314 (2001).

[7] H. Müther and A. Sedrakian, Phys. Rev. Lett. 88, 252503 (2002); Phys. Rev. C 67, 015802 (2003).

[8] M. Alford, J. Berges, and K. Rajagopal, Phys. Rev. Lett. 84, 598 (2000).

[9] M. Alford, J. A. Bowers, and K. Rajagopal, Phys. Rev. D 63, 074016 (2001).

[10] J. A. Bowers and K. Rajagopal, Phys. Rev. D 66, 065002 (2002).

[11] R. Casalbuoni and G. Nardulli, Rev. Mod. Phys. 76, 263 (2004).

[12] H. T. C. Stoof, M. Houbiers, C. A. Sackett, and R. G. Hulet, Phys. Rev. Lett. 76, 10 (1996).

[13] R. Combescot, Europhys. Lett. 55, 150 (2001).

[14] J. Mur-Petit, A. Polls, and H.-J. Schulze, Phys. Lett. A 290, 317 (2001).

[15] W. V. Liu and F. Wilczek, Phys. Rev. Lett. 90, 047002 (2003).
[16] Michael McNeil Forbes, E. Gubankova, W. V. Liu, and F. Wilczek, Phys. Rev. Lett. 94, 017001 (2005).

[17] A. Sedrakian, J. Mur-Petit, A. Polls, and H. Müther, condmat/0404577.

[18] T. Mizushima, K. Machida, and M. Ichioka, Phys. Rev. Lett. 94, 060404 (2005).

[19] A. Sedrakian, J. Mur-Petit, A. Polls, and H. Müther, Phys. Rev. A 72, 013613 (2005).

[20] P. Castorina, M. Grasso, M. Oertel, M. Urban, and D. Zappala, Phys. Rev. A 72, 025601 (2005).

[21] Kun Yang, Phys. Rev. Lett. 95, 218903 (2005); cond-mat/ 0508484; cond-mat/0603190.

[22] J. Dukelsky and G. Ortiz, S. M. A. Rombouts, and K. Van Houcke, Phys. Rev. Lett. 96, 180404 (2006).

[23] M. Mannarelli, G. Nardulli, and M. Ruggieri, Phys. Rev. A 74, 033606 (2006).

[24] L. He, M. Jin, and P.-f. Zhuang, Phys. Rev. B 73, 214527 (2006).

[25] P. F. Bedaque, H. Caldas, and G. Rupak, Phys. Rev. Lett. 91, 247002 (2003); H. Caldas, Phys. Rev. A 69, 063602 (2004); H. Caldas, cond-mat/0601148.

[26] W. Yi and L.-M. Duan, Phys. Rev. A 73, 031604(R) (2006).

[27] E. Gubankova, E. G. Mishchenko, and F. Wilczek, Phys. Rev. Lett. 94, 110402 (2005).

[28] A. Bulgac, M. M. Forbes, and A. Schwenk, Phys. Rev. Lett. 97, 020402 (2006).

[29] F. Chevy, Phys. Rev. Lett. 96, 130401 (2006).

[30] T. N. De Silva and E.J. Mueller, Phys. Rev. A 73, 051602(R) (2006); Phys. Rev. Lett. 97, 070402 (2006).

[31] Kun Yang and S. Sachdev, Phys. Rev. Lett. 96, 187001 (2006); S. Sachdev and Kun Yang, cond-mat/0602032.

[32] E. Gubankova, A. Schmitt, and F. Wilczek, Phys. Rev. B 74, 064505 (2006).

[33] D. T. Son and M. A. Stephanov, Phys. Rev. A 74, 013614 (2006).

[34] P. Pieri and G. C. Strinati, Phys. Rev. Lett. 96, 150404 (2006).

[35] C.-H. Pao, Shin-Tza Wu, and S.-K. Yip, Phys. Rev. B 73, 132506 (2006).

[36] J. Kinast, A. Turlapov, J. E. Thomas, Q. Chen, J. Stajic, and K. Levin, cond-mat/0502087.

[37] A. Sedrakian, T. Alm, and U. Lombardo, Phys. Rev. C 55, R582 (1997).

[38] R. Balian, H. Flocard, and M. Veneroni, nucl-th/9706041; Phys. Rep. 317, 251 (1999).

[39] C.-C. Chien, Q. Chen, Y. He, and K. Levin, Phys. Rev. Lett. 97, 090402 (2006).

[40] L. He, M. Jin, and P.-f. Zhuang, cond-mat/0606322.

[41] K. Fukushima, Phys. Rev. D 72, 074002 (2005).

[42] I. A. Shovkovy, S. B. Ruester, and D. H. Rischke, J. Phys. G 31, S849 (2005) and references therein.

[43] A. Fetter and J.D. Walecka, Quantum Theory of Many Particle Systems (Mc. Graw Hill, Boston, 1971), pg. 459.

[44] A. I. Akhiezer, A. A. Isayev, S. V. Peletminsky, and A. A. Yatsenko, Phys. Rev. C 63, 021304(R) (2001). 